\title{
Radio universality and template-based pulse synthesis
}

\author{
David Butler ${ }^{1,5, \star}$, Tim Huege ${ }^{1,3}$, and Olaf Scholten ${ }^{2,4}$ \\ ${ }^{1}$ IKP, Karlsruher Institut fur Technologie, 76021 Karlsruhe, Germany \\ ${ }^{2} \mathrm{KVI}$ - Center for Advanced Radiation Technology, Zernikelaan 25 NL-9747 AA Groningen, The Netherlands \\ ${ }^{3}$ Astrophysical Institute, Vrije Universiteit Brussel, Pleinlaan 2, 1050 Brussel, Belgium \\ ${ }^{4}$ Interuniversity Institute for High-Energy, Vrije Universiteit Brussel, Pleinlaan 2, 1050 Brussel, Belgium \\ ${ }^{5}$ David Butler acknowledges the support by the DFG-funded Doctoral School "Karlsruhe School of Elemen- \\ tary and Astroparticle Physics: Science and Technology"
}

\begin{abstract}
When discussing radio emission from cosmic ray air showers we commonly make a number of assumptions regarding the production and propagation physics. Incorporating all of these it should be possible to construct a forward model to predict the radio signal produced by an air shower from simple parameters, an application and generalisation of shower universality to radio emission.

In terms of particle detection shower universality focuses on the one-dimensional longitudinal profile, counting only the number of particles. This appears insufficient in the context of radio emission, the particle cascade develops on the scale of traversed atmospheric depth while electromagnetic radiation scales with the geometric trajectories of the sources. Further a real shower extends several radio wavelengths in the lateral direction while analyses often assume a point source on the shower axis.

Our simulation studies show an unanticipated complexity in the radio output responsible for around $10 \%$ variation in the signals. We are still in the process of identifying the relevant quantities and improving our analytical modeling accordingly.
\end{abstract}

\section{Introduction}

To model cosmic ray air showers we have access to Monte Carlo simulation codes encompassing our full knowledge of quantum interactions. These models such as CORSIKA [1] or ZHAireS [2] agree very well when it comes to radio signals generated by the cascade, both with each other and with measurements [3]. These simulations are computationally demanding however and their radio output formats are limited in practical applications. As a complementary approach macroscopic descriptions of the radio production mechanism based on classical electrodynamics have been conceived [4], but fail to reach sufficiently reliable agreement with data.

As an alternative approach we employ CORSIKA-CoREAS [5] radio output sliced by atmospheric depth of origin as templates as outlined in [6].

Our model approximates each atmospheric slice as a point source located on the shower axis, but with a complex angular and temporal emission pattern directly extracted from CoREAS. To first order the far-field radiation is expected to travel along a straight line of sight $l$ with the velocity $c / n_{\text {eff }}(l)$

^e-mail: david.butler@kit.edu 

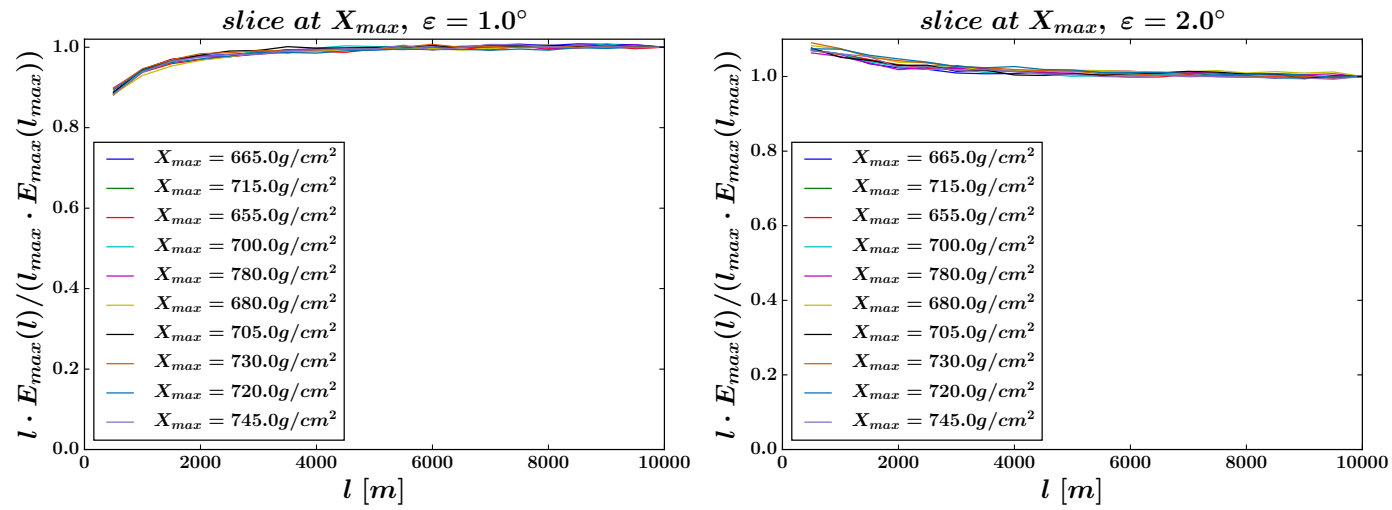

Figure 1. Line-of-sight scaled peak time series amplitude for the $\mathbf{v} \times \mathbf{B}$-polarisation, filtered to $0-500 \mathrm{MHz}$. Ten different simulations with the given $X_{\max }$ values are shown, each rescaled to their respective values at the maximum simulated distance of $10 \mathrm{~km}$. For short distances there is a visible divergence from a point source scaling, which additionally changes sign around the Cherenkov angle of roughly $1.3^{\circ}$.

where $n_{\text {eff }}(l)$ follows the same effective refractive index model as used in CoREAS itself. For a perfect point source the amplitude ought to scale as $1 / l$ which also forms the underlying assumption of the endpoint formalism as applied to individual particle tracks.

\section{Line-of-sight scaling}

Due to technical constraints the slices are larger than $50 \mathrm{~m}$ in the longitudinal direction and have infinite lateral extent, thus some deviation is expected at observation wavelengths as low as $1 \mathrm{~m}$ since the finite size of the source can be resolved. As shown in Fig. (1) divergence from simple $1 / l$ scaling of the pulse amplitude becomes relevant at $<4 \mathrm{~km}$ distance to the source. For the vertical showers considered here this translates to an atmospheric depth of $630 \mathrm{~g} / \mathrm{cm}^{2}$, above typical $X_{\max }$ values for the $10^{17} \mathrm{eV}$ protons used as primary particles. Efforts to model or derive this scaling are ongoing. Fig. (2) shows the corresponding pulse arrival times. Here the agreement is much better and seemingly limited by the numerical resolution of $0.2 n s$ in determining the peak position. As this lies beyond current experimental timing accuracies we focus on improving the amplitude description.

\section{Source strength}

In addition to the absolute number $N$ of electrons and positrons in the source region coherent radio emission also requires these particles to propagate without excessive scattering. A naive model would thus suggest emission strength to scale with the mean free path length which is inversely proportional to the ambient density $\rho$. As the particle number extracted from CORSIKA represents the response of an ideal planar detector an additional scaling with $\rho$ is incurred from properly normalising with the longitudinal slice thickness. We additionally test other physically motivated quantities such as the vectorial average over all electron and positron tracks within each slice representing an effective dipole moment. Using a fixed transmission geometry with equal viewing angles and line-of-sight distances to avoid the additional scaling outlined in Section 2 we can construct an effective longitudinal profile for the radio emission. These profiles are compared in Fig. (3) showing subtle differences between all of them. 

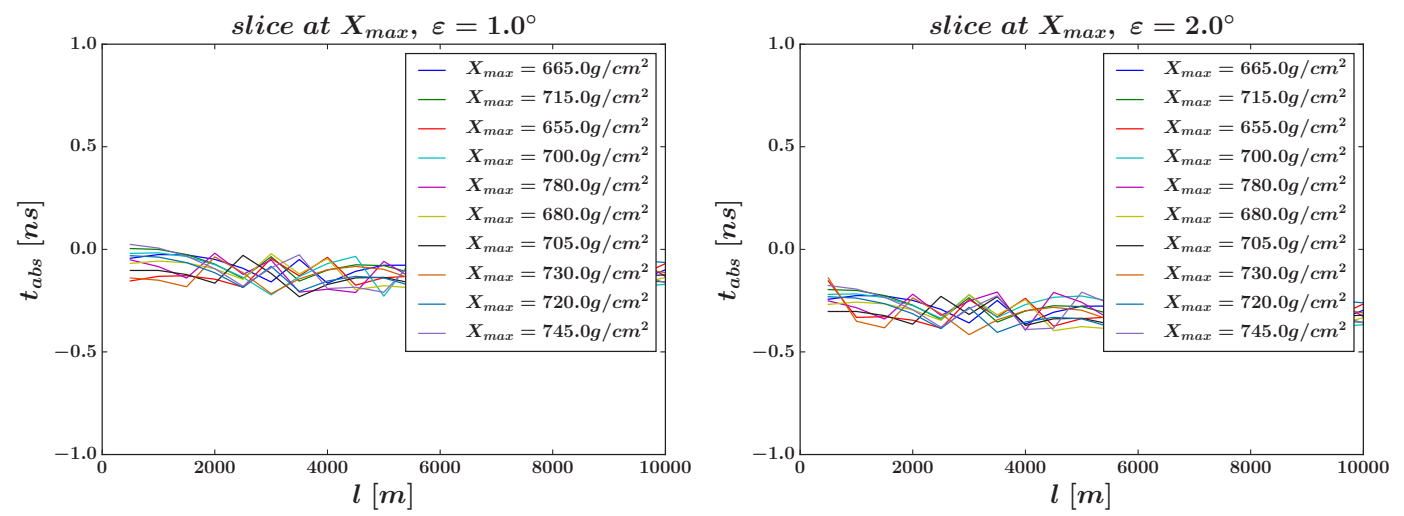

Figure 2. Peak arrival times for the same runs and lines of sight used in Fig. (1), corrected for radio propagation at constant effective velocity $c / n_{\text {eff. }}$. The fluctuations are within the numerical resolution limit of $0.2 \mathrm{~ns}$.

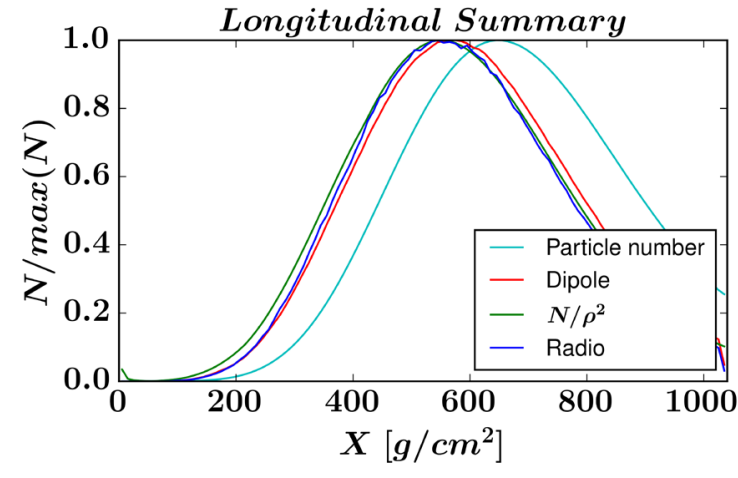

Figure 3. Longitudinal comparison of various quantities. The particle number was extracted directly from CORSIKA. The "dipole" curve was extracted using the COAST interface. The radio profile uses pulses filtered to $0-500 \mathrm{MHz}$ observed at $3.5 \mathrm{~km}$ distance to the slice centre.

\section{Outlook}

Work is still underway to understand the origin of the complex behaviour observed in the radio signal. We also aim to generalise our model to non-vertical showers and varying properties of the primary particle to allow a direct comparison to measured data in a realistic scenario.

\section{References}

[1] D. Heck et al. FZKA Report 6019, (1998)

[2] A. Muñiz et al. Astroparticle Physics 35, 325-341 (2012)

[3] T. Huege, AIP Conference Proceedings 1535, 121-127 (2013)

[4] O. Scholten et al. PRD 97, 023005 (2018)

[5] T. Huege, M. Ludwig, C. W. James, AIP Conference Proceedings 1535, 128-132 (2013)

[6] D. Butler, T. Huege, O. Scholten, PoS ICRC2017, 307 (2017)

[7] S. Lafebre et al. Astroparticle Physics 31, 243-254 (2009) 